\title{
Correction to: New Non-Existence Proofs for Ovoids of Hermitian Polar Spaces and Hyperbolic Quadrics
}

\author{
J. Bamberg, J. De Beule and F. Ihringer®
}

\begin{abstract}
We correct some lemmas in Section 3 and Section 4 in 'New non-existence proofs for ovoids of Hermitian polar spaces and hyperbolic quadrics', Ann. Comb., 21(1):25-42, 2017.
\end{abstract}

Correction to: Ann. Comb. 21 (2017):25-42 https://doi.org/10.1007/s00026-017-0346-0

\section{Corrections for Section 3}

Proposition 3.4, Corollary 3.5, and Lemma 3.8 require that the group $G$ needs to act generously transitively. This does not suffice. All aforementioned results are correct if we demand that $G$ has rank 3. For instance, the following is a correct version of Proposition 3.4.

Proposition 1. Let $G$ be a rank 3 group on $\Gamma$. Let $\chi, \psi \in \mathbb{C}^{n}$ with $\chi, \psi \notin\langle j\rangle$. Then the following statements are equivalent.

(a) One of the vectors $\chi$ and $\psi$ is a non-trivial weighted $m$-ovoid, and one of the vectors is a non-trivial weighted $i$-tight set.

(b) For all $g \in G$, we have $\psi^{g} \chi^{\top}=m i$.

To prove this, we need the following result by Delsarte [3, Th. 6.8].

Theorem 1. Let $G$ be a group which acts generously transitively on $\Gamma$. Let $\chi, \psi \in \mathbb{C}^{n}$. Then the following statements are equivalent.

(a) For all non-principal minimal idempotents $E$ of the association scheme arising from $G$, we have $\chi E=0$ or $\psi E=0$. 
(b) Over all $g \in G$, the scalar product $\psi^{g} \chi^{\top}$ is constant.

Proof. (Proposition 1) As in [1], write $\mathbb{C}^{n}=\langle j\rangle \perp V_{+} \perp V_{-}$. and let $E_{+}$and $E_{-}$be the non-principal idempotents corresponding to $V_{+}$and $V_{-}$. As $G$ is rank 3, there are only two non-principal minimal idempotents in the view of Theorem 1 , that is $E_{+}$and $E_{-}$. So a nontrivial vector of $\langle j\rangle \perp V_{-}$is precisely a weighted $m$-ovoid, and a nontrivial vector of $\langle j\rangle \perp V_{+}$is precisely a weighted $i$-tight set. Suppose (a) holds, and without loss of generality, suppose $\chi$ is a (nontrivial) weighted $m$-ovoid and $\psi$ is a (nontrivial) weighted $i$-tight set. Now every image of $\psi$ under the action of $G$ is also a weighted $i$-tight set, and so by [1, Lemma 3.3], (b) holds. Conversely, if (b) holds, then over all $g \in G$, the scalar product $\psi^{g} \chi^{\top}$ is constant. So by Theorem 1 , we have $\chi E_{+}=0$ or $\psi E_{+}=0$, and $\chi E_{-}=0$ or $\psi E_{-}=0$, where $E_{ \pm}$is the projection to $V_{ \pm}$. By definition, (a) holds.

To see why we need $G$ to be rank 3, consider the following. Suppose that $G$ is rank 4 . Then we have three non-principal minimal idempotents. Denote the corresponding eigenspaces by $V_{1}, V_{2}$ and $V_{3}$. Suppose that $V_{+}=V_{1}$ and $V_{-}=V_{2} \perp V_{3}$. Let $\chi$, respectively, $\psi$ be a non-trivial weighted $m$-ovoid, respectively, $m^{\prime}$-ovoid. Suppose that $\chi \in\langle j\rangle \perp V_{2}$ and $\psi \in\langle j\rangle \perp V_{3}$. Then $\chi^{g} \in\langle j\rangle \perp V_{2}$ for all $g \in G$, so $\psi^{g} \chi^{\top}=m m^{\prime}$ for all $g \in G$, but $\chi$ is not a weighted tight set.

Correction for Sect. 4 Lemma 4.3 is wrong, but not needed. A correct version of our proof of Theorem 4.5 due to Hendrik Van Maldeghem can be found in $[2, \S 2.6]$.

\section{Acknowledgements}

We thank Klaus Metsch for pointing out the mistake in Sect. 3. We thank Hendrik van Maldeghem for pointing out the mistake in Sect. 4.

Publisher's Note Springer Nature remains neutral with regard to jurisdictional claims in published maps and institutional affiliations.

\section{References}

[1] J. Bamberg, J. De Beule, and F. Ihringer. New non-existence proofs for ovoids of Hermitian polar spaces and hyperbolic quadrics. Ann. Comb., 21(1):25-42, 2017.

[2] A. E. Brouwer and H. Van Maldeghem. Strongly Regular Graphs. To Appear. https://homepages.cwi.nl/ aeb/math/srg/rk3/srgw.pdf.

[3] P. Delsarte. Pairs of vectors in the space of an association scheme. Philips Res. Rep., 32(5-6):373-411, 1977. 


\section{J. Bamberg}

Centre for the Mathematics of Symmetry and Computation, Department of Mathematics and Statistics

The University of Western Australia

35 Stirling Highway

Crawley, WA, 6009

Australia

e-mail: John.Bamberg@uwa.edu.au

J. De Beule

Department of Mathematics and Data Science

Vrije Universiteit Brussel

Pleinlaan 2

1050 Brussels

Belgium

e-mail: Jan.De.Beule@vub.be

F. Ihringer

Vakgroep Wiskunde: Analyse, Logica en Discrete Wiskunde Universiteit Gent

Krijgslaan 281

9000 Gent,

Belgium

e-mail: Ferdinand.Ihringer@ugent.be 